\title{
Development and Validation of an In Silico Rabbit Purkinje Cell Action Potential Model: A Step Towards a Drug Safety Testing Tool
}

\author{
Jordi Cano $^{1}$, Julio Gomis-Tena ${ }^{1}$, Alexander Amberg ${ }^{2}$, Lennart Anger ${ }^{2}$, Veronique Ballet ${ }^{3}$, Jean- \\ Michel Guillon ${ }^{3}$, Manuel Pastor ${ }^{4}$, Ferran Sanz ${ }^{4}$, Lucía Romero ${ }^{1}$, Javier Saiz ${ }^{1}$ \\ ${ }^{1}$ Centro de Investigación e Innovación en Bioingeniería (CI2B), Universitat Politècnica de València, \\ Valencia, Spain \\ ${ }^{2}$ SANOFI, R\&D Preclinical Safety, Frankfurt, Germany \\ ${ }^{3}$ SANOFI, R\&D Preclinical Safety, Paris, France \\ ${ }^{4}$ Research Programme on Biomedical Informatics (GRIB), Institut Hospital del Mar d'Investigacions \\ Mèdiques (IMIM), Dept. of Experimental and Health Sciences, Universitat Pompeu Fabra, \\ Barcelona, Spain
}

\begin{abstract}
Companies and regulators evaluate compounds, cardiac safety commonly through in-vitro assays involving cardiac cells. Rabbit cardiac Purkinje Cells (RPCs) are very sensitive to drug effects such as Action Potential Duration (APD) alteration by ion channel block. Our objective is to create a novel RPC model calibrated with the latest experimental data. We developed a new RPC model using Pan-Rudy's Canine Purkinje cell model as a framework. We adapted important parameters, namely, size, ion fluxes and conductances. We also included a sodium current Markovian formulation to account for its recently increased importance. Variables were adjusted to match the main experimentally observed RPCs features. Steady-state was reached by pacing the model for 1500 seconds at different Basic Cycle Lengths (BCLs). The model was validated by extracting data from experimental sources. Results show overall agreement with the literature with improvements to previous models. In conclusion, we improved and successfully validated an RPC model. This work paves the way towards a reliable in-silico tool for testing drug effects on RPCs.
\end{abstract}

\section{Introduction}

In-vitro experiments on several cardiac cell types are commonly used to assess a drug arrhythmogenesis prior to use in man. Nonetheless, since the arrival of the Comprehensive in vitro Proarrhythmia Assay (CiPA) paradigm, in combination to those, mathematical models play now a more important role in the drug-induced proarrhythmic risk assessment[1]. Rabbit Purkinje cells have shown to be very sensitive to Action Potential (AP) disturbance by drugs[2] turning them into a good substrate for drug testing. Recent studies have shown the importance of sodium channels, especially late sodium current $\left(\mathrm{I}_{\mathrm{NaL}}\right)$ in the AP shape[3]. More detailed mathematical descriptions of this current are now being implemented to account for special drug-channel interactions[4], but currently available rabbit Purkinje models lack such formulation.

Our objective is to build a new rabbit Purkinje cell model on top of previously published work while integrating newly available data and dynamics, especially regarding sodium channels. This represents a first step towards an improved proarrhythmic risk assessment tool.

\section{Methods}

\subsection{Reference dataset}

We searched in the literature for studies whose main target was the rabbit cardiac Purkinje cell and gathered data about size, Action Potential Duration (APD), Resting Membrane Potential (RMP), Action Potential Amplitude (APA) and Upstroke Velocity (dV/dt) at different Base Cycle Lengths (BCLs). We selected control assays in drug testing studies. We also digitized the AP shapes to compare our model to traces from literature.

Parameters were obtained as follows: APD $_{90}$ was the time between the moment of maximum upstroke velocity and the $90 \%$ repolarization; RMP was the membrane potential before stimulus was applied, APA was the difference between the maximum value of the potential during depolarization and $\mathrm{dV} / \mathrm{dt}$ was the maximum slope during depolarization. Calcium restitution was measured as the evolution of peak intracellular calcium $\left([\mathrm{Ca}]_{\mathrm{i}}\right)$ as a function of tested BCLs. 


\subsection{Model construction}

We used Pan-Rudy's canine Purkinje cellular model[5], or PRd, as a framework and adjusted all necessary parameters to match the characteristics of a rabbit Purkinje cell, namely size and ion fluxes. Main currents, including $\mathrm{I}_{\mathrm{Kr}}, \mathrm{I}_{\mathrm{Ks}}, \mathrm{I}_{\mathrm{to}}$ and $\mathrm{I}_{\mathrm{K} 1}$ were imported from Corrias model[6]. Additionally, a Markovian formulation of the $\mathrm{I}_{\mathrm{Na}}$ channel from Moreno et al.[7] was introduced. Note that because $\mathrm{I}_{\mathrm{NaL}}$ is also included in these equations, we removed the original current from the model. Calcium dynamics were tweaked to correctly adjust peak intracellular calcium concentrations at different BCLs. Finally, conductances were calibrated to reproduce experimental results found in the literature.

\subsection{Simulations}

We stabilized our model as well as Corrias[6] and Aslanidi et al.[8] models with 1500-second simulations at BCLs of 500, 750, 1000, 2000 and $5000 \mathrm{~ms}$ (called "test BCLs" from now on). Our model was paced with a -80 $\mathrm{pA} / \mathrm{pF}$ stimulus, 1.5 times the depolarization threshold. . Other models were used with their out-of-box parameters, although their code was adapted for convenience. Model results were measured at the last beat of every simulation.

\section{Results}

Table 1. PRd model parameters that have been modified during model calibration. $\mathrm{Gx}$ stands for $\mathrm{x}$ channel conductance; $\mu 1$ is the normal-to-burst transition rate in the Markovian formulation of $\mathrm{I}_{\mathrm{Na}}$; $[\mathrm{Na}$ ] stands for sodium concentrations; $\tau$ are calcium flux time constants; SERCA refers to calcium uptake.

\begin{tabular}{cccc}
\hline Parameter & Value & Parameter & Value \\
\hline $\mathrm{G}_{\mathrm{Kr}}(\mathrm{pA} / \mathrm{pF})$ & 1.566 & {$[\mathrm{Na}](\mathrm{mM})$} & 6.7 \\
$\mathrm{G}_{\mathrm{Ks}}(\mathrm{pA} / \mathrm{pF})$ & 0.0435 & length $(\mu \mathrm{m})$ & 119.76 \\
$\mathrm{G}_{\mathrm{CaL}}(\mathrm{pA} / \mathrm{pF})$ & 5.6 & radius $(\mu \mathrm{m})$ & 13.76 \\
$\mathrm{G}_{\mathrm{Na}}(\mathrm{pA} / \mathrm{pF})$ & 27.5 & $\tau_{\mathrm{gap}}(\mathrm{ms})$ & 4 \\
$\mu 1\left(\mathrm{~ms}^{-1}\right)$ & $4.1 \cdot 10^{-7}$ & $\tau_{\mathrm{ss}}(\mathrm{ms})$ & 0.05 \\
$\mathrm{G}_{\mathrm{K} 1}(\mathrm{pA} / \mathrm{pF})$ & 35 & $\tau_{\text {tr } 1}(\mathrm{~ms})$ & 40 \\
$\mathrm{G}_{\text {to fast }}(\mathrm{pA} / \mathrm{pF})$ & 5 & $\tau_{\text {tr } 2}(\mathrm{~ms})$ & 40 \\
$\mathrm{G}_{\text {to slow }}(\mathrm{pA} / \mathrm{pF})$ & 1.2 & $\mathrm{SERCA}$ & $+10 \%$ \\
\hline
\end{tabular}

Table 1 summarizes all parameters that we modified in order to reproduce our reference dataset. We reduced ion flux time constants to account for the new cell size, but we maintained cell compartments as in PRd. We adjusted $\mathrm{G}_{\mathrm{Kr}}$, $\mathrm{G}_{\mathrm{CaL}}, \mathrm{G}_{\mathrm{Na}}, \mathrm{G}_{\text {to fast }}$ and $\mathrm{G}_{\text {to slow }}$ to correctly adjust current peaks, $\mathrm{APD}_{90}$ restitution curves and plateau potential. We imported sodium concentrations from Corrias model.
Figure 1 shows the AP waveforms of the model at test BCLs. The plateau phase duration increases with greater BCLs, mostly due to an increase in late sodium current $\mathrm{I}_{\mathrm{NaL}}$ (the "late" portion of $\mathrm{I}_{\mathrm{Na}}$ ). The final model showed APD $90 \mathrm{~S}$ of 229.9, 266.2, 290.9, 344.8 and $395.2 \mathrm{~ms}$; APA were $124.1,127.5,129,129.3$ and $130 \mathrm{mV}$; RMP were -84.78 , $84.72,-84.63,-84.25$ and $-83.65 \mathrm{mV}$; and finally, $\mathrm{dV} / \mathrm{dt}$ were $472.1,422.3,409.5,535.5$ and $496.7 \mathrm{~V} / \mathrm{s}$, respectively, at test BCLs, as can be seen in Figure 2.
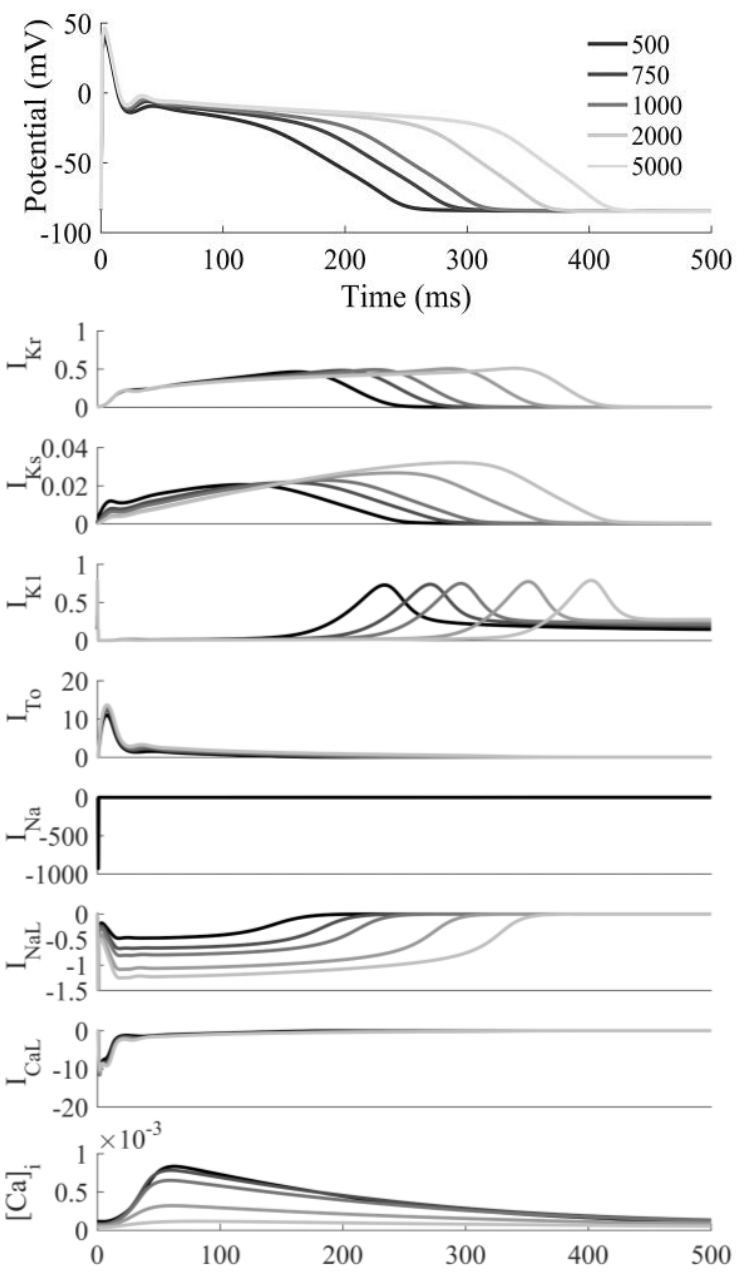

Figure 1. Action Potentials (top), main currents (in $\mathrm{pA} / \mathrm{pF}$ ) and intracellular calcium transients (bottom, in $\mathrm{mM}$ ) from 1500-second simulations last beat at test BCLs. Note that $\mathrm{I}_{\mathrm{NaL}}$ is a rescaled trace of $\mathrm{I}_{\mathrm{Na}}$.

Our model correctly reproduces the reference data, which was mostly present for BCL $1000 \mathrm{~ms}$. We found a scarcity of data for the rest of the BCLs including some contradictory results for RMP at BCL 500 and $750 \mathrm{~ms}$, as well as for $\mathrm{dV} / \mathrm{dt}$, whose range could reach as low as around $300 \mathrm{~V} / \mathrm{s}$ and as high as over $700 \mathrm{~V} / \mathrm{s}$. 

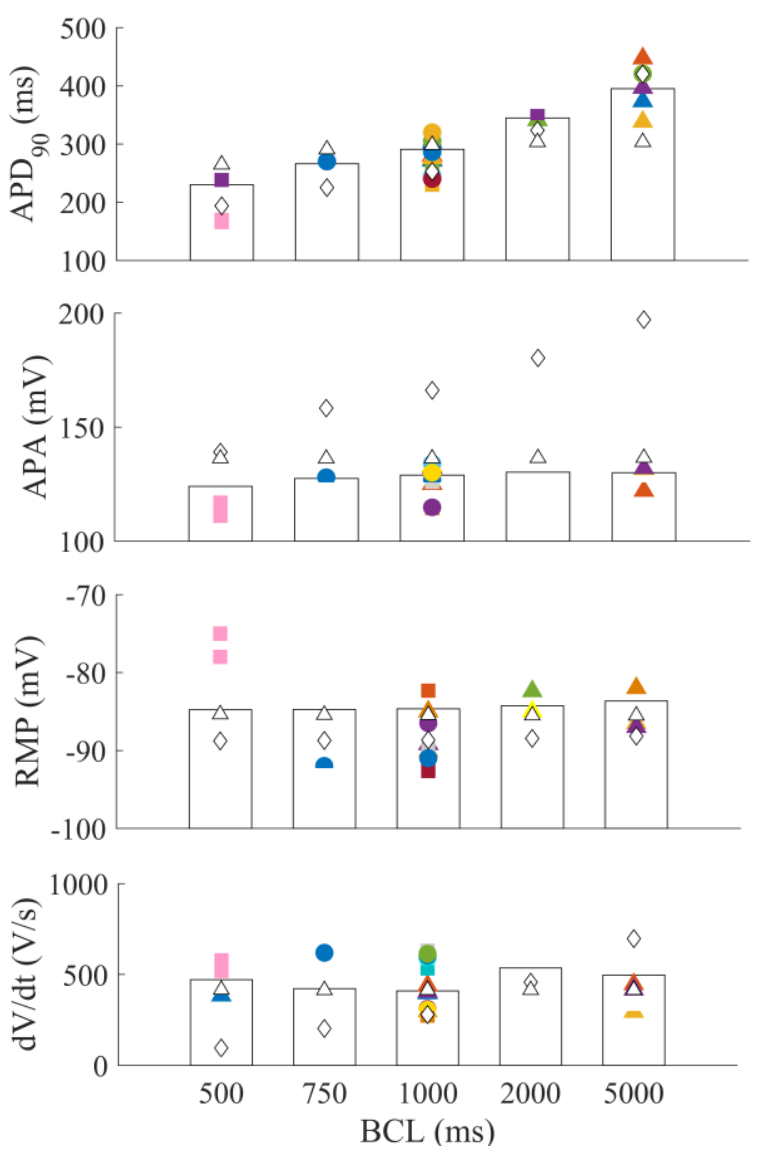

Figure 2. Bar plots of the parameters of our model compared to experimental references found in literature[9,10,19-23,11-18] (colored symbols) including internal data from Sanofi, Aslanidi et al.[8] (white triangles) and Corrias[6] (white diamonds) models.

Simulations from other models were also plotted in Figure 2. On the one hand, compared to Aslanidi et al.[8] model simulations, our model provides an improvement of the $\mathrm{APD}_{90}$ restitution at high $\mathrm{BCLs}$, with a minor improvement on the APA.

On the other hand, compared to Corrias[6] model simulations, our model seems to perform equally considering $\mathrm{APD}_{90}$ and RMP but, since the former is highly dependent on APA, these values might not represent the actual restitution curve. In contrast, our model shows a more stable APA and RMP which are key to good APD 90 values.

Figure 3 depicts normalized to maximum peak calcium concentrations of our model compared to digitized data from Schmidt et al.[24]. Our model shows, calcium peaks of $94 \%, 78 \%$ and $38 \%$ compared to reference $85 \%, 75 \%$ and $47 \%$ at BCL 750, 1000 and $2000 \mathrm{~ms}$ respectively. Values were kept close to the original Corrias[6] model.

Figure 4 shows our model's AP shape at BCL $1000 \mathrm{~ms}$ on top of digitized traces from available references found in literature. Overall, AP waveforms seem to agree with it, despite the high variability of the reference experimental data. As for plateau potentials and repolarization, APs appear as though as our model could fit as an average trace.

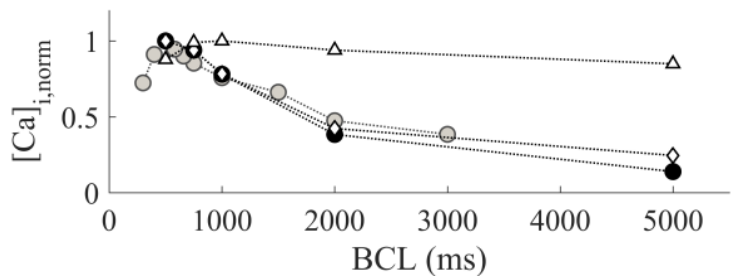

Figure 3. Peak calcium concentrations plotted against BCL. Normalized results from our model (black dots) reference from Schmidt et al. 1998 (grey dots), Aslanidi et al. (white triangles) and Corrias (white diamonds) models.

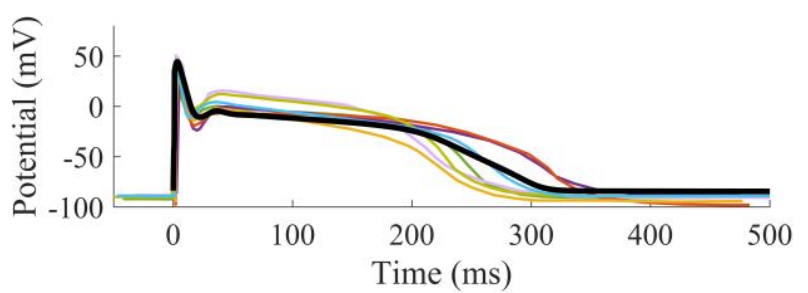

Figure 4. Representation of our model's AP at BCL 1000 ms (black trace) compared to several digitized APs found in literature $[2,15,25,26]$ including internal data from Sanofi.

\section{Conclusions}

We have built a new rabbit Purkinje cell AP model by combining previous work with new data and experiments. Our model correctly reproduces reference features from literature in control simulations at five different BCL.

Recent findings have raised the importance of sodium channels in cardiac cells. Our model includes a more detailed formulation of the late sodium current[7].

A recent study of rabbit Purkinje cell AP restitution shows that AP shape highly depends on $\mathrm{I}_{\mathrm{NaL}}$, a feature that could be the reason behind a wider range of AP durations[3]. Our model is in agreement because of its ability to adapt to slower pace rates, correctly adjusting its $\mathrm{APD}_{90}$. This is our first iteration on the RPC model which we will improve in a future work.

\section{Acknowledgements}

This work was partially supported by grants from the Ministerio de Economía y Competitividad and Fondo Europeo de Desarrollo Regional (FEDER) DPI201569125-R (MINECO/FEDER, UE) and from the "Programa Prometeo", Generalitat Valenciana, Award Number: 2016/088. 


\section{References}

[1]. Fermini B, Hancox JC, Abi-Gerges N, Bridgland-Taylor $\mathrm{M}$, Chaudhary KW, Colatsky T, et al. A new perspective in the field of cardiac safety testing through the comprehensive in vitro proarrhythmia assay paradigm. J Biomol Screen. 2016 Jan 1;21(1):1-11.

[2]. Lu HR, Vlaminckx E, Gallacher DJ. Choice of cardiac tissue in vitro plays an important role in assessing the risk of drug-induced cardiac arrhythmias in human: beyond QT prolongation. J Pharmacol Toxicol Methods. 2008;57(1):18.

[3]. Li W, Yu Y, Hou J-W, Zhou Z-W, Guo K, Zhang P-P, et al. Larger rate dependence of late sodium current in cardiac Purkinje cells: A potential link to arrhythmogenesis. Hear Rhythm. 2017;14(3):422-31.

[4]. Moreno JD, Lewis TJ, Clancy CE. Parameterization for insilico modeling of ion channel interactions with drugs. Bondarenko VE, editor. PLoS One. 2016 Mar 10;11(3):e0150761.

[5]. Li P, Rudy Y. A model of canine purkinje cell electrophysiology and ca2+ cycling: rate dependence, triggered activity, and comparison to ventricular myocytes. Circ Res. 2011 Jun 24;109(1):71-9.

[6]. Corrias A, Giles W, Rodriguez B. Ionic mechanisms of electrophysiological properties and repolarization abnormalities in rabbit Purkinje fibers. Am J Physiol Heart Circ Physiol. 2011 May;300(5):H1806-13.

[7]. Moreno JD, Yang P-C, Bankston JR, Grandi E, Bers DM, Kass RS, et al. Ranolazine for congenital and acquired late INa-linked arrhythmias: in silico pharmacological screening. Circ Res. 2013 Sep 13;113(7):e50-61.

[8]. Aslanidi O V., Sleiman RN, Boyett MR, Hancox JC, Zhang $\mathrm{H}$. Ionic mechanisms for electrical heterogeneity between rabbit purkinje fiber and ventricular cells. Biophys J. 2010 Jun;98(11):2420-31.

[9]. Aubert M, Osterwalder R, Wagner B, Parrilla I, Cavero I, Doessegger L, et al. Evaluation of the rabbit Purkinje fibre assay as an in vitro tool for assessing the risk of druginduced torsades de pointes in humans. Drug Saf. 2006;29(3):237-54.

[10]. Conlon K, De Maeyer JH, Bruce C, Schuurkes JAJ, Christie L, McRedmond J, et al. Nonclinical cardiovascular studies of prucalopride, a highly selective 5hydroxytryptamine 4 receptor agonist. J Pharmacol Exp Ther. 2018 Feb [cited 2018 Apr 13];364(2):156-69.

[11]. Ducroq J, Printemps R, Le Grand M. Additive effects of ziprasidone and d,l-sotalol on the action potential in rabbit Purkinje fibres and on the hERG potassium current. J Pharmacol Toxicol Methods. 2005 Jul;52(1):115-22.

[12]. Ducroq J, Printemps R, Guilbot S, Gardette J, Salvetat C, Le Grand M. Action potential experiments complete hERG assay and QT-interval measurements in cardiac preclinical studies. J Pharmacol Toxicol Methods. 2007 Sep;56(2):159-70.

[13]. Dumaine R, Cordeiro JM. Comparison of $\mathrm{K}+$ currents in cardiac Purkinje cells isolated from rabbit and dog. J Mol Cell Cardiol. 2007 Feb;42(2):378-89.

[14]. Gluais P, Bastide M, Caron J, Adamantidis M. Risperidone prolongs cardiac action potential through reduction of $\mathrm{K}+$ currents in rabbit myocytes. Eur J Pharmacol. 2002 May;444(3):123-32.
[15]. Goineau S, Castagné V, Guillaume P, Froget G. The comparative sensitivity of three in vitro safety pharmacology models for the detection of lidocaineinduced cardiac effects. J Pharmacol Toxicol Methods. $2012 \mathrm{Jul} ; 66(1): 52-8$.

[16]. Hof T, Sallé L, Coulbault L, Richer R, Alexandre J, Rouet $\mathrm{R}$, et al. TRPM4 non-selective cation channels influence action potentials in rabbit Purkinje fibres. J Physiol. 2016 Jan 15;594(2):295-306.

[17]. Lee H-A, Hyun S-A, Park S-G, Kim K-S, Kim SJ. Comparison of electrophysiological effects of calcium channel blockers on cardiac repolarization. Korean J Physiol Pharmacol. 2016;20(1):119.

[18]. Lu HR, Vlaminckx E, Van Ammel K, De Clerck F. Druginduced long QT in isolated rabbit Purkinje fibers: importance of action potential duration, triangulation and early afterdepolarizations. Eur J Pharmacol. 2002 Oct;452(2):183-92.

[19]. Roche M, Renauleaud C, Ballet V, Doubovetzky M, Guillon J-M. The isolated rabbit heart and Purkinje fibers as models for identifying proarrhythmic liability. J Pharmacol Toxicol Methods. 2010 May;61(3):238-50.

[20]. Cordeiro JM, Spitzer KW, Giles WR. Repolarizing K+ currents in rabbit heart Purkinje cells. J Physiol. 1998;508(3):811-23.

[21]. Lu HR, Vlaminckx E, Teisman A, Gallacher DJ. Choice of cardiac tissue plays an important role in the evaluation of drug-induced prolongation of the QT interval in vitro in rabbit. J Pharmacol Toxicol Methods. 2005;52(1):90-105.

[22]. Yan D, Cheng L, Song H-Y, Turdi S, Kerram P. Electrophysiological effects of haloperidol on isolated rabbit Purkinje fibers and guinea pigs papillary muscles under normal and simulated ischemia. Acta Pharmacol Sin. 2007 Aug;28(8):1155-60.

[23]. Masumiya H, Saito M, Ito M, Matsuda T, Noguchi K, IidaTanaka N, et al. Lack of action potential-prolonging effect of terfenadine on rabbit myocardial tissue preparations. Biol Pharm Bull. 2004;27(1):131-5.

[24]. Schmidt U, Hajjar RJ, Helm P a, Kim CS, Doye a a, Gwathmey JK. Contribution of abnormal sarcoplasmic reticulum ATPase activity to systolic and diastolic dysfunction in human heart failure. J Mol Cell Cardiol. 1998;30(10):1929-37.

[25]. Lu HR, Vlaminckx E, Van de Water A, Gallacher DJ. Calmodulin antagonist W-7 prevents sparfloxacin-induced early afterdepolarizations (EADs) in isolated rabbit purkinje fibers: importance of beat-to-beat instability of the repolarization. J Cardiovasc Electrophysiol. 2006 Apr;17(4):415-22.

[26]. Lu HR, Vlaminckx E, Cools F, Gallacher DJ. Direct effects of arsenic trioxide on action potentials in isolated cardiac tissues: Importance of the choice of species, type of cardiac tissue and perfusion time. J Pharmacol Toxicol Methods. 2012 Sep;66(2):135-44.

\section{jsaiz@ci2b.upv.es}

Javier Saiz

Universitat Politècnica de València: Camino de Vera s/n, 46022, Valencia, Spain. 